\section{Vorteile für laparoskopische Operation beim Rektumkarzinom}

\author{
Minimalinvasive Eingriffe werden auch beim Rektumkarzinom immer \\ beliebter. In einer großen Studie haben Chirurgen nun geprüft, ob \\ laparoskopische Eingriffe bei Rektumkarzinomen tatsächlich ver- \\ gleichbar gute Ergebnisse liefern wie die offene Operation.
}

—ür die randomisiert-kontrollierte tal cancer Laparoscopic or Open Resection II) haben europäische Chirurgen aus acht Ländern insgesamt 739 Patienten mit Rektumkarzinom minimalinvasiv operiert, 364 wurden einer klassischen offenen Operation unterzogen. Geeignet waren Patienten, bei denen keine Hinweise auf Fernmetastasen vorlagen und bei denen sich der Tumor nicht weiter als $15 \mathrm{~cm}$ vom Anus entfernt befand. Die Chirurgen sollten den Tumor samt mesorektalem Gewebe entfernen und zugleich die hypogastrischen Nerven schonen.

Primärer Endpunkt war die Rezidivrate nach drei Jahren - diese Daten liegen allerdings noch nicht vor. Aber im- merhin konnten die Studienautoren schon einmal die Daten zu einigen sekundären Endpunkten auswerten. Einer davon war die Rate makroskopisch kompletter Resektionen: Sie war mit $88 \%$ in der laparoskopischen Gruppe nicht signifikant schlechter als in der Gruppe mit offener Operation (92\%). Keine Unterschiede gab es zudem bei der Rate mikroskopisch erfolgreicher Resektionen. Eine solche lag vor, wenn in den äußeren $2 \mathrm{~mm}$ des resezierten Gewebes keine Tumorzellen mehr aufzuspüren waren. Dies war in beiden Gruppen bei $90 \%$ der Patienten der Fall.

Wie zu erwarten, gab es bei der minimalinvasiven Operation einen geringeren Blutverlust (im Median 200 vs. 400 $\mathrm{ml}$ ), die Darmfunktion kam schneller wieder in Gang (zwei vs. drei Tage) und die Patienten konnten schneller nach Hause entlassen werden (nach acht vs. neun Tagen). Bei der Sterberate und der Rate postoperativer Komplikationen gab es jedoch keine signifikanten Differenzen. Einziger Nachteil der laparoskopischen Operation: Sie dauerte deutlich länger (vier vs. drei Stunden).

Fazit: Mit dieser Studie konnte gezeigt werden, dass die Tumorresektion unter Alltagsbedingungen in Kliniken laparoskopisch ebenso gründlich erfolgt wie bei der offenen Operation. Dies war in der Vergangenheit immer wieder bezweifelt worden.

Die Patienten erholen sich zudem schneller, verlieren weniger Blut und kommen früher aus der Klinik, wenn sie minimalinvasiv operiert werden. Allerdings ist die Komplikationsrate dabei nicht unbedingt geringer als bei einer offenen Operation.

Thomas Müller

van der Pas MHGM et al. Laparoscopic versus open surgery for rectal cancer (COLOR II): shortterm outcomes of a randomised, phase 3 trial. Lancet Oncol. 2013;14(3):210-8.

\title{
CRC: Zwei Inhibitoren des VEGF im Vergleich
}

\section{Nach den ESMO-Guidelines von 2010 gehört der Anti-VEGF-A-Antikörper Bevacizumab in Kombination mit den FOLFOX/FOLFIRI-Regimes zum Standard in der Erstlinientherapie des kolorektalen Karzinoms (CRC). Die Erweiterung der Oxaliplatin/Fluoropyrimidin-basierten Chemotherapie um den VEGF-Rezeptorhemmer führte zwar zu signifikant verlängerten progressionsfreien Krankheitsverläufen, verbesserte aber nicht das Gesamtüberleben der Patienten.}

n einer Phase-I-Studie mit unbehandel-
ten, an einem metastasierten CRC er-
krankten Patienten erwies sich der ora-
len VEGF-Tyrosinkinaseinhibitor Cedi-
ranib kombiniert mit dem mFOLFOX6-
Regime als gut verträglich und wirksam.
Gestützt auf diese Ergebnisse wurde die
doppelblinde, randomisierte Phase-II/
III-Studie HORIZON III initiiert, in der
Cediranib plus mFOLFOX6 mit der
Kombination Bevacizumab plus
mFOLFOX6 verglichen wurde. Primärer
Endpunkt der Studie war das progressi-
onsfreie Überleben (PFS). Im Phase-III-
Abschnitt der Studie erhielten 709 Pati- enten täglich oral $20 \mathrm{mg}$ Cediranib plus mFOLFOX6 (85 mg/m ${ }^{2}$ Oxaliplatin und $400 \mathrm{mg} / \mathrm{m}^{2}$ Folinsäure i. v., gefolgt von $400 \mathrm{mg} / \mathrm{m}^{2}$ FU i.v. am ersten Tag und anschließend $2.400 \mathrm{mg} / \mathrm{m}^{2}$ kontinuierlich über die nächsten 46 Stunden in zweiwöchigen Intervallen). 713 Patienten im Vergleichsarm erhielten anstelle des VEGF-Tyrosinkinaseinhibitors $50 \mathrm{mg} / \mathrm{kg}$ Bevacizumab alle 14 Tage.

Erste Analysen ergaben keine signifikanten Unterschiede zwischen beiden Gruppen bezüglich des progressionsfreien Überlebens (median 9,9 Monate un- ter Cediranib vs. 10,3 Monate unter Bevacizumab), des Gesamtüberlebens (22,8 vs. 21,3 Monate) und der Responserate. Das gesetzte Ziel, die statistische Nicht-Unterlegenheit von Cediranib im Hinblick auf das progressionsfreie Überleben wurde nicht erreicht.

In der Therapiebeurteilung aus Patientensicht schnitt die Kombination mit Cediranib zudem schlechter ab als das Vergleichsregime.

Fazit: Bei der Behandlung des metastasierten kolorektalen Karzinoms ist der VEGF-Tyrosinkinaseinhibitor Cediranib in Kombination mit mFOLFOX6 ähnlich aktiv wie Bevacizumab, erhielt aber aufgrund seines Nebenwirkungsprofils eine schlechtere Beurteilung seitens der Patienten. Wolfgang Zimmermann

Schmoll HJ et al. Cediranib with mFOLFOX6 versus bevacizumab with mFOLFOX6 as first-line treatment for patients with advanced colorectal cancer: a double-blind, randomized phase III study (HORIZON III). J Clin Oncol.

2012;30(29):3588-95. 